\title{
Physical Modelling and Control of Dynamic Foaming in an LD-Converter Process
}

\author{
Wolfgang Birk ${ }^{\star \dagger}$, Ioannis Arvanitidis ${ }^{\ddagger}$, Pär Jönsson ${ }^{\ddagger}$ and Alexander Medvedev ${ }^{\dagger}$ \\ *Corresponding author: Tel. +46920 91907,Fax +46920 91558, eMail wolfgang@sm.luth.se \\ ${ }^{\dagger}$ Control Engineering Group, Luleå University of Technology, SE-971 87 Luleå, Sweden \\ $\ddagger$ Department of Metallurgy, Royal Institute of Technology, SE-100 44 Stockholm, Sweden
}

\begin{abstract}
This paper deals with physical modelling and control of dynamic foaming in the LD-converter process. An experimental setup consisting of a water model, DSP and PC hardware is built up and showed to be useful for studying dynamic foaming. Furthermore, a foam height estimation algorithm is presented and validated through experiments. Finally, sound signals from the LD-converter and water model are compared and similarities between them are found.
\end{abstract}

\section{INTRODUCTION}

Steel is widely produced from hot metal and scrap. Hot metal with about $4-5 \% C$ is produced from iron oxide pellets and coke by the blast furnace. After the blast furnace, the hot metal is poured into a ladle and usually converted into steel by a top blown basic oxygen furnace (BOF). Oxygen is supplied through a lance from above and is jetted onto the metal bath at supersonic speed. Scrap, slag forming agents and hot metal are charged to the vessel and, thereafter, the lance is lowered with oxygen blown through. The oxygen jet forms a cavity at the bath surface where $F e$, $S i, M n$ and $C$ are oxidised. Metal droplets are splashed and mixed together with the slag. Carbon dissolved in the metal phase reacts with $\mathrm{FeO}$ to form carbon monoxide:

$$
\mathrm{C}+\mathrm{FeO}=\mathrm{Fe}+\mathrm{CO}(\mathrm{g})
$$

This means that a foam containing metal droplets, metal oxides (slag) and $C O$ bubbles is formed. The effective surface area of this foam is large and thereby the chemical reactions involved in this process are fast. To make this process effective, a large foam volume is needed. However, if the foam level is too high, slopping occurs causing reduction in metal exchange and environmental pollution. The formation of the foam is manually controlled by adjusting the oxygen lance level. The foam level in the vessel is nearly impossible to measure, but there has been somewhat successful methods using radio waves and sonic meters. Optical methods based on laser technology have been tried with bad results because of the strong dust formation during the foaming. A very simple and continuous method to estimate foam level is to use a microphone and measure the sound intensity at certain frequency bands. As the foam level increases, the sound intensity decreases. Microphones or sonic meters have been widely used since 1970 in many steel plants. However, the sound signal generated is seldom used in order to automatically control the foam level. Piombino Steel shop, ILVA Taranto and British steel are some examples where the sound signal is used as a control signal [1], [2], [3]. In [4], the sonic meter signal is estimated from off-gas flow rate and $C O$ content in the gas. There, the deviation of estimated signal from measured signal is used to detect slopping. The purpose of the present work is to gain more insight in control aspects, using the sound signal, and to get a better understanding of foaming.

First, a background on foaming and its relation to the converter process is given. In the second part of Section II foam height estimation and control of the foam height are discussed. Next, the experimental setup is described in Section III. In the following section the performed experiments are presented. Section $V$ shows the application of the foam height estimation algorithm on experimental data and gives a comparison of the water model with the LD-converter in respect to recorded sound data. Finally, Section VI summarizes the results and gives an outlook on the future work.

\section{BACKGROUND}

First preliminaries on foaming are given and it is shown how dynamic foaming is related to foaming in the $L D$ converter process. Furthermore, it is explained why a water model can be used to resemble the LD-converter process. Based on the knowledge on sound propagation through foam, a methodology for foam height estimation is derived. Finally, it is discussed how the estimate can be used for control of the foam height.

\section{A. Foaming}

A foam usually consists of a gas phase distributed as bubbles in a liquid phase. Gas could either be formed by a chemical reaction in the liquid phase or by injection of an external gas below the liquid surface. Foaming is generally affected by viscosity, surface tension, bubble size and gas generation rate. A foam can not be produced from a pure liquid. Surface active elements which lower the surface tension must be added in order to create a foam. The more a surface active element lowers the surface tension, the more stable foam is formed. Hence it is more important to study the change of surface tension than its absolute value. Room temperature experiments, [5], showed an increase in 
steady state foam heights with increased surface tension depression.

Foam life, $\tau$ after stopping the gas injection, could be determined by measuring foaming height, $h(t)$, as function of time. The following relationship has been found [6], [7]:

$$
\tau=-\frac{t}{\ln \left(\frac{h(t)}{h(0)}\right)}
$$

Foam life increases with increasing viscosity of the liquid but decreases with increasing flow rate of injected gas. The height of the foam increases with increased gas flow rate.

According to [6], [7], the foaming index is defined as:

$$
\Sigma=\frac{h}{u_{g}}
$$

where $h$ is the foam height and $u_{g}$ is the superficial gas velocity. With $\alpha$ as the gas fraction, the superficial gas velocity is given by:

$$
u_{g}=\frac{u_{S}}{\alpha},
$$

where $u_{S}=$ (gas flow rate) $/$ (section area of vessel). The steady state foam height increases with increased superficial gas velocity up to a critical value of $u_{g}$ and starts thereafter to decrease with increased $u_{g}$. This breakdown and collapse of the foam is caused by turbulence in the system [5]. The foaming index has the dimension of time and is the average gas travel time through the foamed layer. Foaming index is dependent of the liquid phase density, $\rho$, gravity, $g$, gas bubble diameter, $d_{b}$, viscosity, $\mu$, and the difference of surface tension between pure liquid and the solution, $\Delta \sigma . H$. G. Lee et al [8] derived with dimensional analysis the following equation:

$$
\frac{\Sigma \Delta \sigma}{\mu d_{b}}=k\left\{\frac{\Delta \sigma}{\rho g d_{b}^{2}}\right\}^{\delta}
$$

According to (4), foaming index increases with increasing viscosity, $\mu$, and hence the foam height increases with $\mu$ as well.

\section{B. Foaming in the oxygen steel making converters}

A mixture of liquid slag, metal droplets and gas bubbles (emulsification or foam), is formed when the oxygen jet hits the metal bath in the BOF. The foam is formed by the oxygen gas impact and penetration of the liquid steel as well as the chemical reaction with oxygen and carbon in the metal bath and the metal droplets,

$$
\frac{1}{2} \mathrm{O}_{2}(g)+\mathrm{C}=\mathrm{CO}(g)
$$

Oxygen is blown with supersonic speed through a water cooled lance. The lance is submerged in the foam but the lance tip is always above the surface of the metal bath. The oxidation rate of carbon is almost constant during about $30 \%$ and $70 \%$ of the blow time. The gas void fraction is estimated to be around $0.5 \ldots 0.95$ by using the ratio between slag volume and available converter volume [9]. Metal droplets splash up from the melt and fall down again through the foam. As the metal droplets are resident, carbon dissolved in the metal phase reacts with the slag to form $C O$ gas which increases the foam volume. A physical room temperature model would be very difficult to design in order to represent the BOF by keeping dimensionless numbers such as Reynolds number or Weber number. Therefore, a model is constructed with the view point on foam level and automatic control.

\section{Foam height estimation}

During foaming the foam height in the converter has to be maximized, whilst slopping has to be avoided. Since the foam height is not directly measurable, because of the working environment, an estimation methodology has to be derived. Available measurements are off-gas analysis and sound recordings from a sonic meter which is placed in an air-floated area in the exhaust fume pipes.

The main sound source is the nozzle of the oxygen lance, where oxygen exits the lance with above sound speed and impinges the metal bath surface. The created foam then acts like a sound absorber and reduces the sound intensity. Hence, the sound intensity can be related to the foam height. According to [10], the sound intensity is attenuated exponentially by the foam:

$$
I(h(t), \omega)=I_{0} e^{-\beta_{F}(\omega) h(t)},
$$

The frequency dependent attenuation coefficient $\beta_{F}(\omega)$ directly depends on the foam characteristics, i.e. bubble size and material. Applying the logarithm, (5) can be rewritten to

$$
h(t)=\frac{\ln \left(I_{0}\right)-\ln (I(h(t), \omega))}{\beta_{F}(\omega)}
$$

Consequently, the foam height should be linear to the logarithm of the intensity of the recorded sound at certain selected frequencies. Estimation of the foam height requires estimation of the attenuation coefficient $\beta_{F}$. The estimate is denoted $\hat{\beta}_{F}$. Thus (6) has to be reformulated in order to give an expression for the estimate

$$
\hat{h}(t, \omega)=\frac{\ln \left(I_{0}\right)-\ln (I(h(t), \omega))}{\hat{\beta}_{F}(\omega)}
$$

The frequency dependency of the estimate can be resolved by combining all estimates over frequency $\omega$. Using a weighted least-squares algorithm the foam height estimate $\hat{h}(t)$ can be obtained. Naturally, frequencies that do not show attenuation of the sound intensity as well as frequencies which mainly contain noise should be neglected 


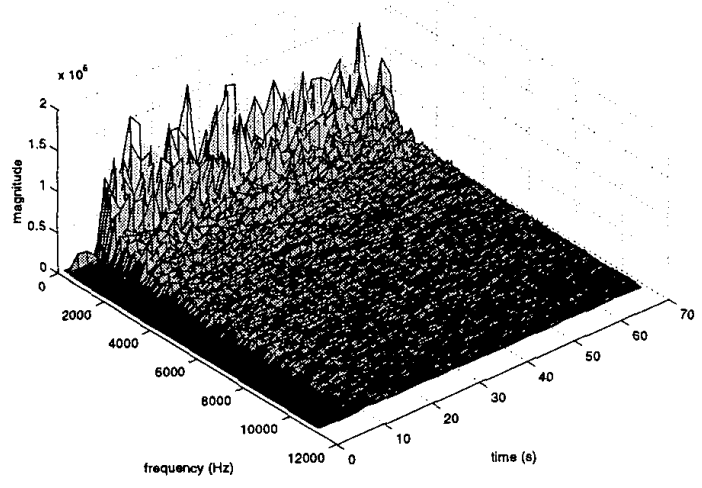

Fig. 1. Example for the spectrum of the recorded sound

or weighted with zero. Eventually, an estimate over time is found.

Fig. 1 shows an example for the sound spectrum recorded during an experiment. Clearly, the parameter $\beta_{F}$ varies over frequency and hence has to be identified for the chosen frequency ranges. Important for the chosen frequencies is the sensitivity of the sound intensity to height changes. In the interesting foam height range the sound intensity should be highly sensitive to small height changes.

Since bubble size and material content cannot be described by physical relationships, it is difficult to derive a first principal model for the foam dynamics. System identification can be used to identify the parameter $\hat{\beta}_{F}$ from experiments. The input signal to the foam is the sound source and the output signal is the sound recorded by the microphone. In this arrangement the microphone dynamics and dynamics of the channel from the foam surface to the microphone are neglected.

Since the sound source cannot be measured at its generation point, the input signal of the identification experiment is unknown. During a foaming experiment there are two characteristic moments, where the recorded sound is directly related to a known foam height:

1. In the beginning of the foaming experiment, the foam height is zero. Hence, $I_{0}$ is recorded.

2. When foam is generated and it just covers the nozzle of the lance, the foam height is equal to the lance height over the fluid. Here, $I\left(h_{L}\right)$ is recorded. All estimates based on this sound have to be biased with the lance height $h_{L}$.

Assuming, both moments are detectable, and the air flow through the lance is constant, the input signal for the identification experiment is known and identification can be applied.

\section{Control of foam height}

The availability of a foam height estimate allows closed loop control of the foam height using the lance position and air flows to lance and bottom as control signals. For this purpose a model should be available that relates the foam height to the given actuator signals.

Presently, no first principal models exist that relate the lance movement to changes in the foam height. Hence, experiments have to be performed first in order to study the behaviour of the foam height provided changes in the actuators. Both static and dynamic experiments should be considered.

Moreover, it should be kept in mind that the behaviour of the water model has to be compared with the behaviour of the LD-converter. Thereby, the water model can be improved and more knowledge of the process can be gained.

\section{EXPERIMENTAL SETUP}

The setup consists of water model, where the foaming takes place, an interface to the DSP and PC Hardware, which processes the arising data, and the software which is in fact part of the DSP and the PC.

\section{A. Water model}

A physical model of the LD reactor is built from a transparent plastic tube, Fig. 2, Fig. 3. In order to study the sound moderation by the foam, the height of the tube $(1 \mathrm{~m})$ was chosen large compared to the inner diameter, $193 \mathrm{~mm}$. The tube was mounted vertically in a steel rig, fixed only at the lower end by four $M 8$ bolts through a disk attached at the bottom of the tube. A lance, supplied with up to $0.6 \mathrm{MPa}$ air, was mounted on a linear actuator attached to the top of the rig. The microphone of condenser type was placed $200 \mathrm{~mm}$ above the opening of the tube and mounted on a stand with no contact with the steel rig in order to avoid vibration from the foaming process.

Two lance nozzles were used, one and five hole. The five hole nozzle has $1.5 \mathrm{~mm}$ diameter holes with $15^{\circ}$ angle to the direction of the lance. The other nozzle has a $1.5 \mathrm{~mm}$ diameter hole in the same direction as the lance.

\section{B. DSP and PC Hardware}

An essential part of the experimental setup is DSP and $\mathrm{PC}$ hardware, as high computational performance is needed for data processing. Selection of the computer hardware has been focused on flexibility and reliability of the structure. A preliminary analysis of the problem has shown that the MCX-DSP board from Precision MicroDynamics Inc. provides sufficiently many inputs and outputs (analogue, digital) and encugh computational performance. The board offers the following features:

- 814 bit analog inputs

- 1614 bit analog outputs

- 96 digital inputs

- 32 digital outputs

- Floating point DSP with 120MFLOP

As host computer for the MCX-DSP board a PC with Windows NT as operating system is chosen. Since only 


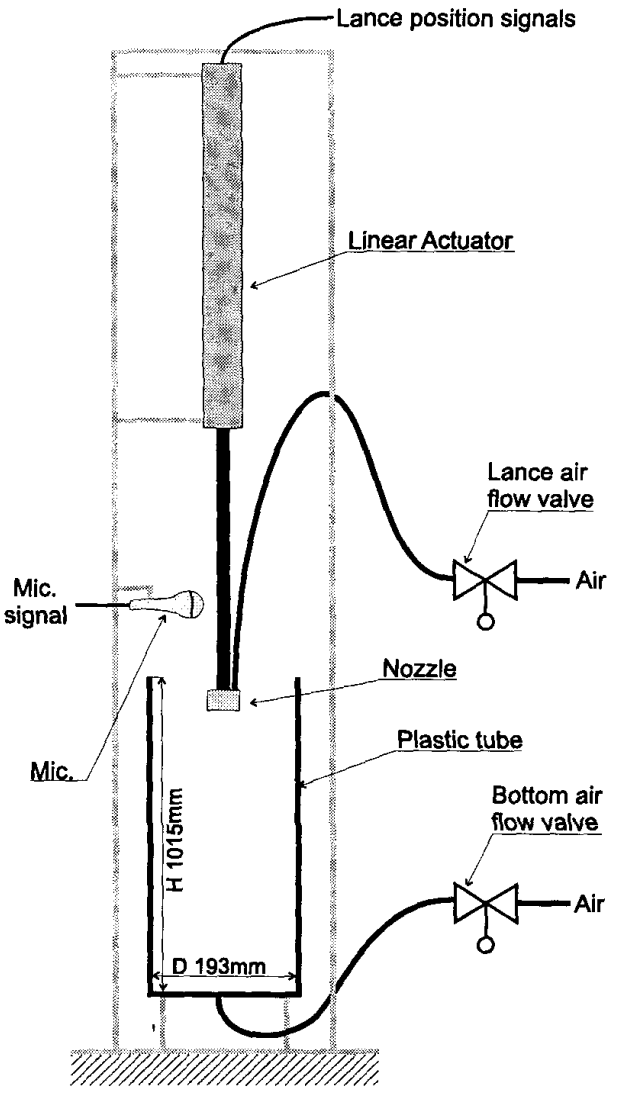

Fig. 2. Schematic drawing of the water model

standard hardware is needed, only the following requirements have been setup:

- At least $256 \mathrm{Mb}$ RAM

- At least $10 \mathrm{~Gb}$ hard disk space

- Direct access to internet via Ethernet card

Since the project is conducted by several parties from different regions of Sweden, the computer used for the experiments has to work as an FTP server. Hence, all participants can instantly access experimental data via Internet and design new algorithms and upload to the experimental setup. Admittedly, it should be avoided to allow access during foaming experiments.

\section{Software and signal flow}

The MCX-DSP board is used to perform control and data acquisition with up to $100 \mathrm{kHz}$ sampling frequency. Since the board has no direct access to any resources in the host computer, a host application has to be in place to create an interface between the board and the hosts resources. Moreover, the $M C X-D S P$ board has no user interface. Thus, user control on the DSP should propagate

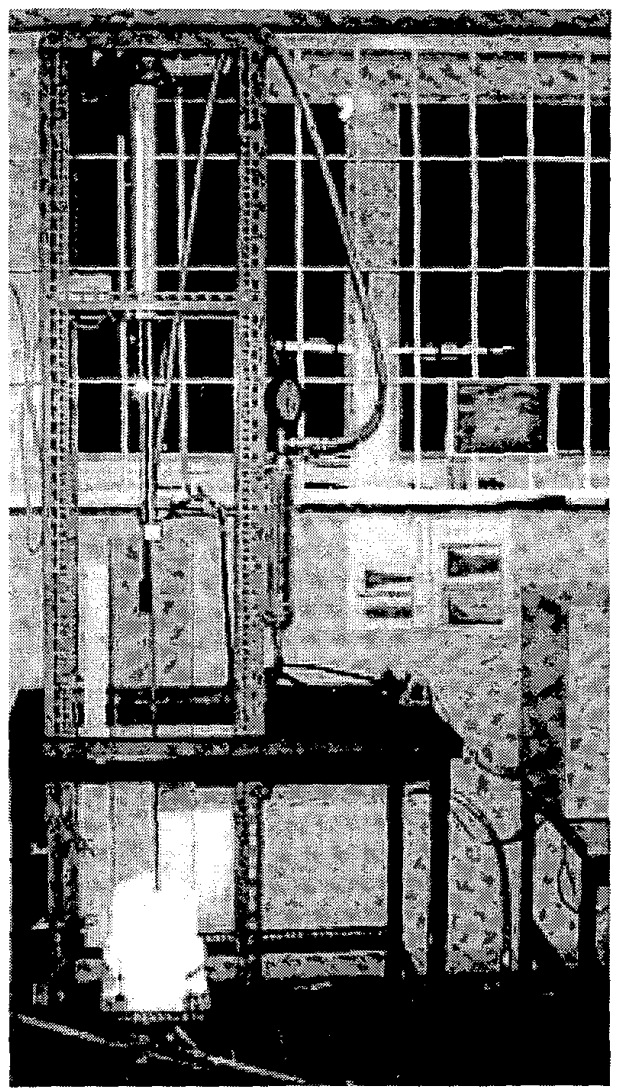

Fig. 3. Photography of the water model

via the host application to the DSP. The general structure of the software setup and the signal flow is depicted in Fig. 4.

Two main difficulties have to be overcome, when designing the software for the experimental setup. First, the real-time synchronisation of host application, control (data acquisition) algorithm and analog/digital converters has to be set up. Since the best timer resolution is found on the DSP ( $25 n s$ ), the synchronisation should be performed by the DSP. Therefore, the DSP application initiates A/D conversion and also raises interrupt signals to Windows NT. Since Windows NT is not a real-time operating system and consequently runs asynchronous to the DSP buffered data transfer from the DSP to the host application has to be implemented.

Second difficulty is the restricted transfer rate via the ISA bus. Technical staff at Precision MicroDynamics Inc. indicated that the transfer rate via the ISA bus using Windows NT can be as low as $500 \mathrm{kbit} / \mathrm{s}$. This, of course, depends on usage of the ISA bus for other applications. Consequently, it should be avoided to run application that 


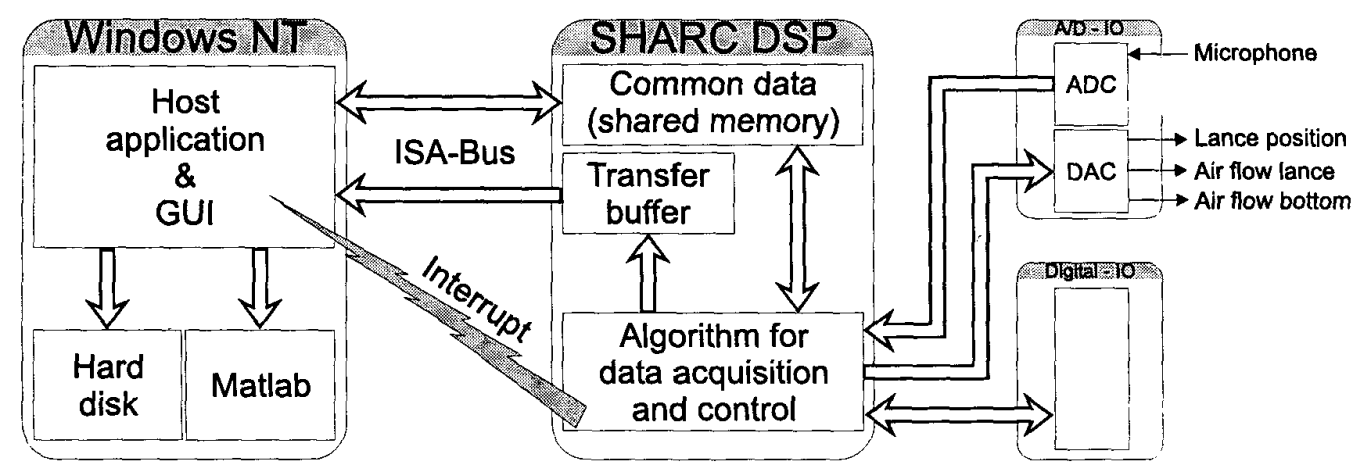

Fig. 4. Structure of the software and signal flow for the experimental setup

use the ISA bus at the same time. It has been indicated to us that data buffering for about $10 \mathrm{~ms}$ on the DSP should avoid data loss.

As mentioned above the control and data acquisition algorithm is run in real-time at a certain sampling frequency. During each cycle the algorithm initiates an $A / D$ conversion and retrieves the data from the converters. Furthermore, digital inputs are read as well. The processed data that should be transferred to the host computer is then placed in the transfer buffer. When a packet of at least 128 data entries is stored, the DSP raises an interrupt signal to Windows NT. When Windows NT catches the interrupt signal it transmits it to the host application, which in turn initiates a data transfer from the DSP to the host. The data is then placed in a large memory buffer. Not until the experiment is finished, the buffered data is flushed to the hard disk.

Using shared memory common data like status and commands are available in the common data area. Both DSP application and host application can access this memory area, which is placed on the DSP. Using this memory area DSP and host application can exchange information and commands. For example, the sampling rate of the algorithm can thereby be adjusted by the user via the host application. It should be pointed out, that the access to this memory area is not subject to any synchronisation.

Tests of the data acquisition algorithm have shown that sampling rates of at least $60 \mathrm{kHz}$ can be achieved with no data losses.

For analysis and evaluation of the experiments Matlab is used. For this purpose a function set has been created that facilitates data import and processing.

\section{EXPERIMENTS}

The used experiment fluid was water mixed with surfactants and glycerol. Four litres of fluid was pored into the tube. Table I displays the specification for the different fluids.

Two types of experiments were carried out, static and
TABLE I

FLUIDS SPECIFICATION

\begin{tabular}{|c|c|c|c|}
\hline Mixture & $\begin{array}{c}\text { Viscosity } \\
{[\mathrm{Cp}]}\end{array}$ & $\begin{array}{c}\text { Foarn life } \tau \\
{[\mathrm{s}]}\end{array}$ & $\begin{array}{c}\text { Surface tension } \\
{[\mathrm{N} / \mathrm{m}]}\end{array}$ \\
\hline \hline $\mathrm{A}$ & 1.0 & 1064 & 0.27 \\
\hline $\mathrm{B}$ & 3.1 & 3448 & 0.27 \\
\hline $\mathrm{C}$ & 1.0 & 189 & 0.49 \\
\hline
\end{tabular}

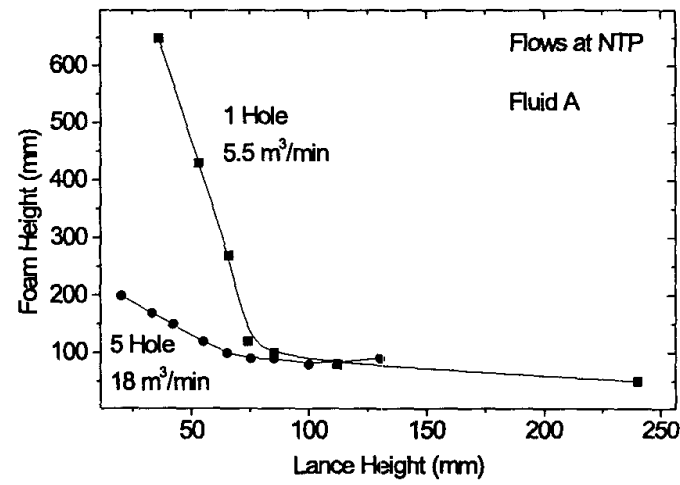

Fig. 5. Results from static foaming of mixture A with different nozzles

dynamic. Static experiments were conducted with a foaming at different lance positions with constant airflow. At each lance position, enough time was elapsed until the foam height did not change. In the dynamic experiments, the foam height was measured as the foam volume was increasing. It should be pointed out that the lance height and the foam height were measured relative to the initial water level before blowing. There will be a certain cavity increasing the true lance height and also some part of the four litres fluid forming the foam is resulting in an underestimation of the foam height.

Fig. 5 shows the static foaming from both one-hole and five-hole nozzle with mixture A. There is a transition from low foam height to high foam height at about $70 \ldots 80 \mathrm{~mm}$ lance height. This transition could be the result of the air 


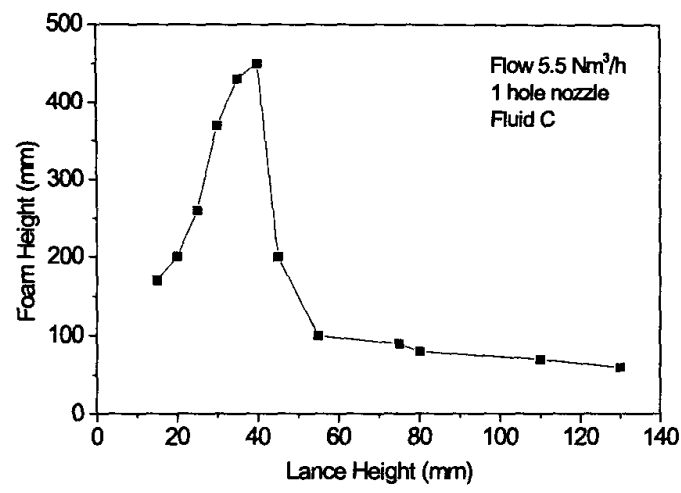

Fig. 6. Results from static foaming of mixture $C$

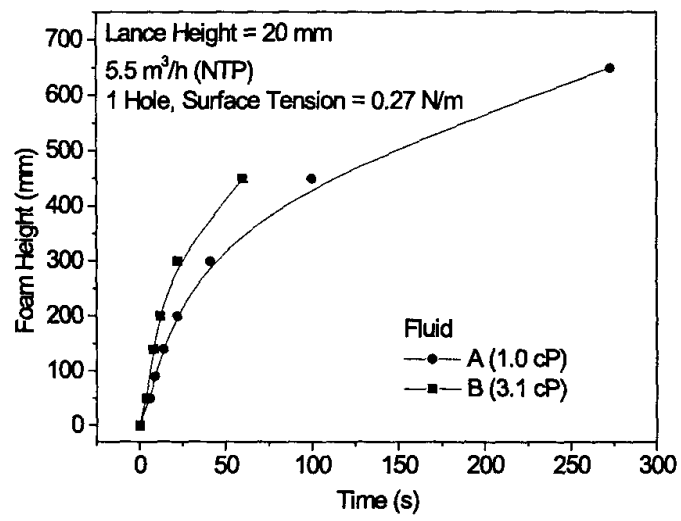

Fig. 7. Results of dynamic foaming of mixture A and B

jet penetrating the water surface. The closer the jet is to the surface, the smaller the diameter of the jet is and, therefore, the larger pressure is exerted onto the surface, making the air to penetrate rather than bounce on the surface.

Fig. 6 shows another static experiment with one hole nozzle with mixture $C$. There is a transition from weak foaming to strong foaming at $55 \mathrm{~mm}$ lance height. The difference in transition points could be explained by the higher surface tension for mixture $\mathrm{C}$ which should predict a higher pressure of the jet in order to penetrate the surface meaning a smaller lance height. There is also a maximum foam height and then the foam height decreases with decreasing lance height. This decrease could be the result of foam degeneration as the jet zone becomes more turbulent at small lance heights.

Fig. 7 show the dynamic foaming as function of time. The experiment was conducted with the lance height constant at $20 \mathrm{~mm}$. The mixture with higher viscosity shows faster foaming.

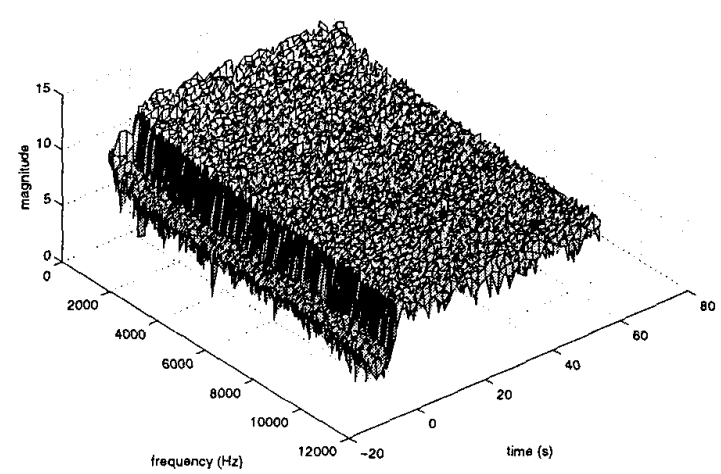

Fig. 8. Logarithm of the sound spectrum for the foaming experiment

\section{SOUND ANALYSIS}

In order to apply the above described estimation scheme, the recorded sound has to be analysed. For the sake of simplicity, the sound spectrum over time is derived by windowing the recorded sound file and then applying the Fourier transform on the windowed data. Fig. 1 shows an example for the obtained spectrum.

Since the foam height is presently not measured automatically, the foam heights are measured manually and are time stamped.

Following the guidelines from above, the sound source is taken from the beginning of the foaming experiment. The moment of occurrence in the recorded sound file is given by the maximum of the intensity. Then, the logarithm is applied to the obtained spectrum, see Fig. 8, and subtracted from logarithm of the spectrum of the sound source.

First, the interesting frequency range has to be chosen. Obviously, all frequencies above $2 \mathrm{kHz}$ are attenuated by the foam, see Fig. 1. The attenuation coefficient can be computed in a least-squares sense and becomes $\hat{\beta}_{F}=600$. By applying the above described estimation methodology to the frequency range between two and $11 \mathrm{kHz}$ yields a foam height estimation over time. Fig. 9 shows a comparison of the measured foam height with the estimated foam height. Clearly, the estimated foam height follows the measured foam height well. Moreover, it has to be pointed out that no filtering has been applied to measurement data or estimate.

Yet, it has to be investigated whether the estimation algorithm can be applied to the LD-converter process. Therefore, sound data of the sonic meter at SSAB Oxelösund's LD-converter has been logged. The spectrum of the sound data has been derived analogously as before and has been zoomed in order to display the interesting region, see Fig. 10.

During the logging of the data all interaction with the 


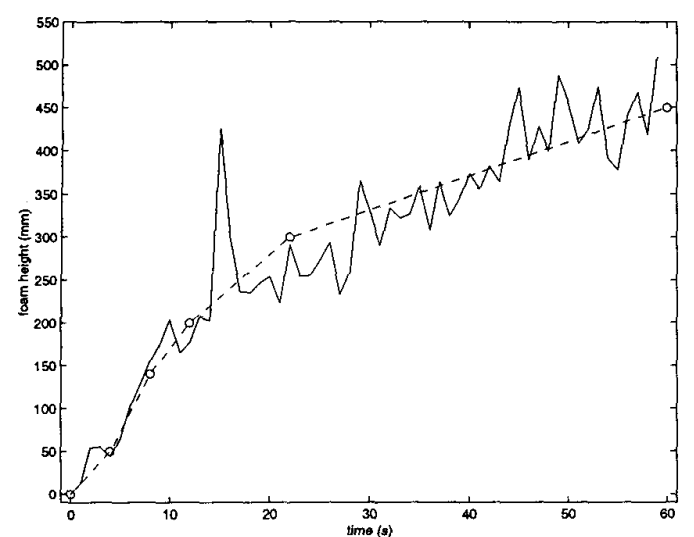

Fig. 9. Estimated foam height (solid line) versus manually measured foam height (circles, dashed line).

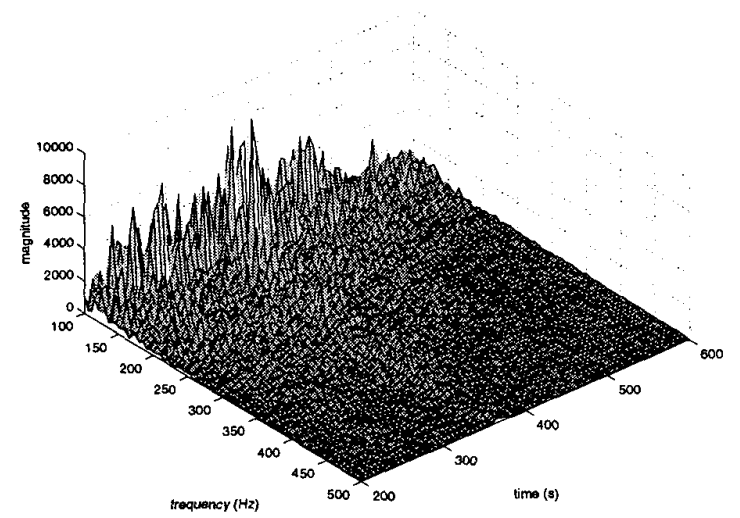

Fig. 10. spectrum of the recorded sound from SSAB Oxelösund's LD-converter

process has been recorded and time stamped. In order to compare water model sound spectrum with LD-converter sound spectrum it is essential to know when the oxygen has been switched on and when the lance is lowered into the vessel. These actions occurred at $250 \mathrm{~s}$ and $320 \mathrm{~s}$, respectively.

Obviously, the maximum sound intensity is reached at about $350 \mathrm{~s}$ which is approximately the time when the lance reached its position. Eventually, the foaming started at this moment. After $350 \mathrm{~s}$ it can be observed, that the sound intensities are attenuated. Thus, the LD-converter reveals a similar behaviour as the water model. Still, the frequency ranges where the phenomena can be observed are different, which is caused by the difference in the material characteristics. Hence, the attenuation coefficient $\beta_{F}$ has to be larger for the LD-converter process.

\section{Conclusions \& Future work}

The theoretical background of foaming is presented and an experimental setup to study dynamic foaming has been built up. Furthermore, an algorithm for foam height estimation based on sound has been developed. Experiments with the water model are performed and show that it is possible to create fluid mixtures to achieve desired foaming characteristics. Using experimental data, the foam height estimation algorithm could be validated. Consequently, control of the foam height can be pursued now. Finally, sound signals from the water model and LD-converter are compared and similarities could be observed.

Notwithstanding these good results, more research has to be conducted. The experimental setup needs to be updated with an automatic foam height measurement, as the quality of the estimation algorithm depends on the quality of the validation data. Furthermore, it has to be investigated how attenuation characteristics of the foam can be brought closer to the characteristics of the LD-converter. Finally, control of the foam height has to be implemented.

\section{ACKNOWLEDGMENTS}

The authors want to thank SSAB Oxelösund $\mathrm{AB}$ for making plant data available, and the students of the project course SMD086 Group D at Luleå University of Technology for their efforts in designing a software interface for the DSP board. Financial support of the Swedish National Board for Industrial and Technical Development (NUTEK) is also gratefully acknowledged.

\section{REFERENCES}

[1] C. Bencini and A. Poli, "Automation for refining and slag control in ld process at AFP/Piombino Steel Shop," in Steelmaking Conference Proceedings, pp. 193-198, 1993.

[2] A. Abbatangelo, M. D. Rena, M. Palchetti, and L. Zampetti, "Blowing pattern computerised control at Taranto Steelshop," in Steelmaking Conference Proceedings, pp. 337-342, 1990

[3] D. Anderson, C. M. Barnes, and H. J. Whittaker, "Fully dynamic process control of the BOS in British Steel," in Steelmak ing Conference Proceedings, pp. 379-387, 1991

[4] D. Widlund, A. Medvedev, and R. Gyllenram, "Towards modelbased closed-loop control of the basic oxygen steelmaking process," in Proc. of the 9th IFAC Symposium on Automation in Mining, Mineral and Metal Processing 1998, Cologne, Germany, 1-3 September 1998, pp. 69-74, 1998.

[5] S. Ghag, P. Hayes, and H.-G. Lee, "Physical model studies on slag foaming," ISIJ International, vol. 38, no. 11, pp. 1201-1207, 1998.

[6] K. Ito and R. Fruehan, "Study on the foaming of $\mathrm{CaO}-\mathrm{SiO} 2-\mathrm{FeO}$ slags: Part i. foaming parameters and experimental results," Met. Trans. B, vol. 20B, pp. 509-514, 1989.

[7] K. Ito and R. Fruehan, "Study on the foaming of $\mathrm{CaO}-\mathrm{SiO} 2$ FeO slags: Part ii. dimensional analysis and foaming in iron and steelmaking processes," Met. Trans. B, vol. 20B, pp. 515-521, 1989.

[8] S. Ghag, P. Hayes, and H.-G. Lee, "Model development of slag foaming," ISIJ International, vol. 38, no. 11, pp. 1208-1215, 1998.

[9] P. Misra, B. Deo, and R. Chabra, "Dynamic model of slag foaming in oxygen steelmaking converters," ISIJ International, vol. 38 , no. 11 , pp. $1225-1232,1998$.

[10] U. Ingard, Notes on Sound Absorption Technology. Noise Control Foundation, 94-02 ed., 1994. 